\title{
Lessons of the month: A forgotten classic: Delayed diagnosis of mitral stenosis presenting initially as Ortner's syndrome
}

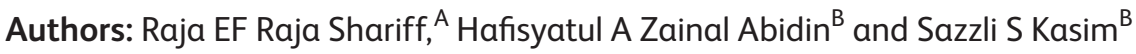

\begin{abstract}
We present a rare case of delayed diagnosis of mitral stenosis, initially presenting with hoarseness in her voice due to left recurrent laryngeal nerve (LRLN) compression. A 60-year-old woman presented to the otorhinolaryngology department following complaints of progressive hoarseness in voice over a 6-month period. There was dysphonia but no additional evidence of a cranial nerve IX or X palsy on examination, with subsequent flexible nasopharyngolaryngoscopy demonstrating left vocal cord palsy. She was referred for a cardiology consult following findings of atrial fibrillation on electrocardiography. Transthoracic echocardiography revealed an enlarged left atrium with evidence of severe mitral stenosis. A diagnosis of Ortner's syndrome was made and the patient underwent mitral valve replacement. Common causes of Ortner's syndrome include mitral stenosis with left atrium compression of the LRLN, but it can occur due to other causes including pulmonary hypertension or aortic aneurysm compression, among others. There are few data at present to conclude that regression of left atrial enlargement and pulmonary arterial hypertension with symptoms are associated with Ortner's syndrome. Therefore, it remains pertinent for clinicians to be aware of clinical features linked to mitral stenosis including its more uncommon presentations, such as in our case, as earlier intervention may improve prognosis.
\end{abstract}

KEYWORDS: Mitral stenosis, Ortner's syndrome, case report

DOI: $10.7861 /$ clinmed.2020-0750

\section{Introduction}

Common clinical presentation of mitral stenosis includes dyspnoea, chest pain and haemoptysis. Rarely, symptoms due to compression of an enlarged left atrium onto neighbouring structures can occur. ${ }^{1}$ We present a rare case of delayed diagnosis of mitral stenosis, initially presenting with hoarseness in voice due to left recurrent laryngeal nerve (LRLN) compression.

Authors: ${ }^{\text {A }}$ cardiology fellow and clinical lecturer, Universiti Teknologi MARA, Sungai Buloh, Malaysia; ${ }^{\text {B }}$ senior clinical lecturer, Universiti Teknologi MARA, Sungai Buloh, Malaysia

\section{Case presentation}

A 60-year-old woman presented to the otorhinolaryngology department following complaints of progressive hoarseness in her voice over a 6 -month period. She was otherwise well with no known medical illnesses prior to presentation. Examination revealed an irregular pulse with rate of 82 beats per minute, and blood pressure of $103 / 82 \mathrm{mmHg}$. Cardiovascular examination revealed a loud mid-diastolic murmur (grade 4/6) with concomitant pansystolic murmur (grade $5 / 6$ ), both louder on expiration. There were no carotid bruits audible. Neurological examination failed to demonstrate signs of upper motor neuron lesions. There was dysphonia but no additional evidence of a cranial nerve IX or X palsy demonstrated.

Flexible nasopharyngolayngoscopy was performed, which revealed a left vocal cord palsy (Fig 1). Magnetic resonance imaging of the brain did not reveal any evidence of cerebral infarctions. She was referred for a cardiology consult following findings of atrial fibrillation (AF) on electrocardiography, which was otherwise unknown prior to admission. Chest X-ray revealed cardiomegaly, with clear lung fields. Transthoracic echocardiography revealed an enlarged left atrium with evidence of severe mitral stenosis, likely rheumatic in aetiology, with evidence of severe mitral regurgitation secondary to mitral annular dilatation (Fig 2). A diagnosis of Ortner's syndrome was made and a cardiothoracic consult was sought. The patient underwent mitral valve replacement and was later commenced on warfarin for her AF and metallic valve prosthesis.

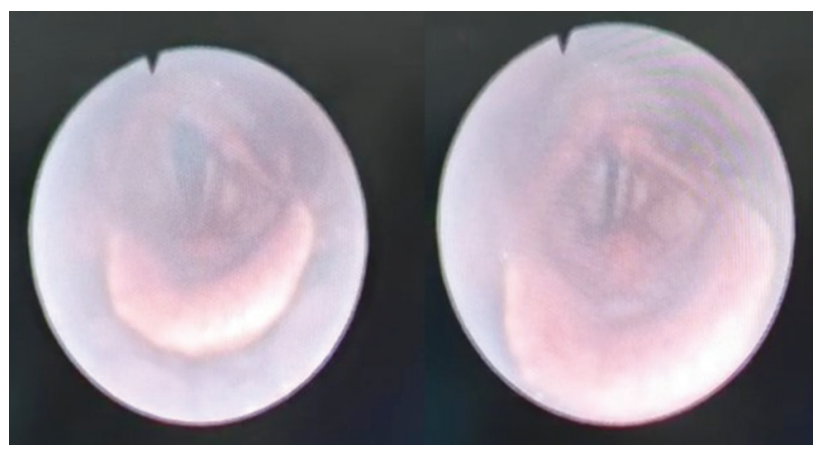

Fig 1. Flexible nasopharyngolayngoscopy revealing a left vocal cord palsy. 
Fig 2. Investigations revealing an enlarged left atrium with evidence of severe mitral stenosis and severe mitral regurgitation secondary to mitral annular dilation. a) Transthoracic echocardiography (TTE) in parasternal long-axis in diastole. b) TTE in parasternal long-axis in systole. c) TTE in apical four-chamber in diastole. d) TTE in apical four-chamber in systole. e) Continuous wave Doppler assessment. f) TTE comparable with Doppler. g) TTE showing mitral annulus diameter of $5.9 \mathrm{~cm}$ and vena contracta measurement of $0.9 \mathrm{~cm}$.
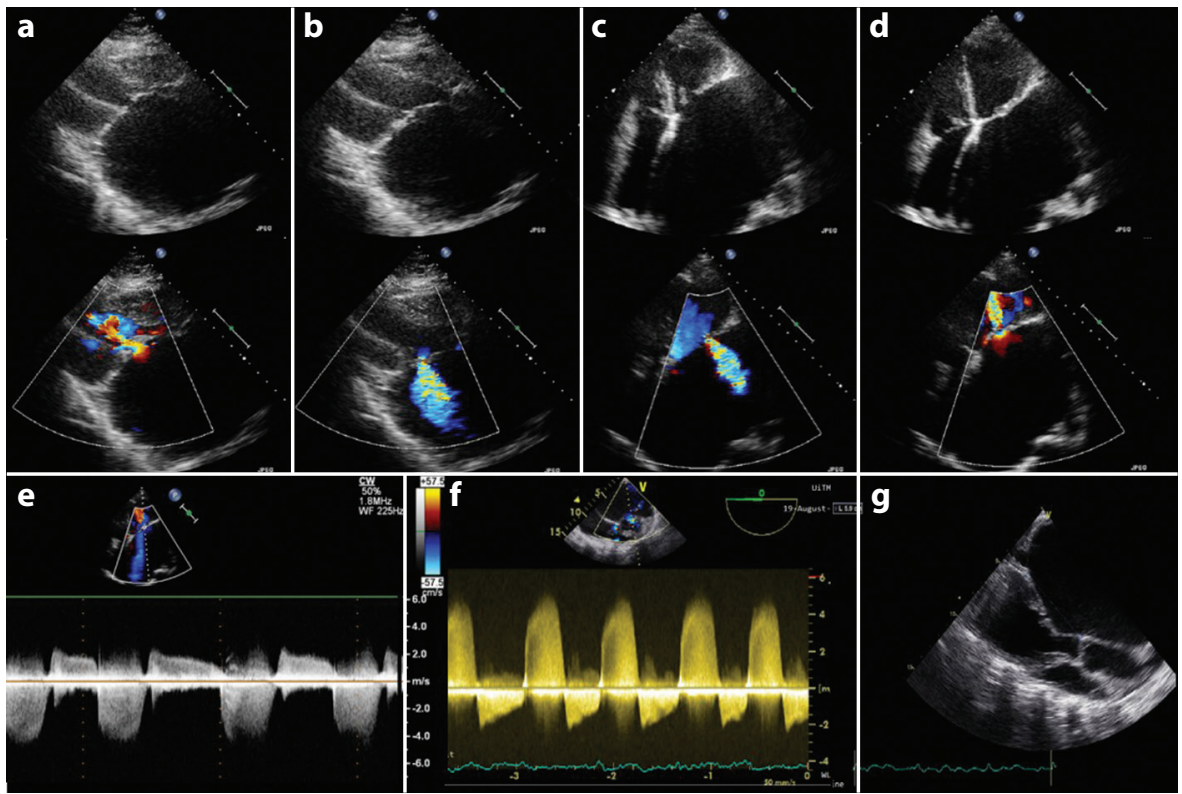

\section{Discussion}

Ortner syndrome, or cardiovocal syndrome, was first described in 1897 in a case series, but remains a rare entity. ${ }^{2}$ Common causes include mitral stenosis with left atrium compression of the LRLN, but it can occur due to other causes including pulmonary hypertension or aortic aneurysm compression, among others. ${ }^{3-5}$ Although rheumatic mitral valve disease is now less commonly found in developed nations, it remains an issue in developing countries like Malaysia. In fact, with rapid globalisation, it may face a resurgence even in developed countries. ${ }^{6}$

It should be noted that partial or gradual compression of the LRLN may not necessarily cause dysphonia and clinicians should remain vigilant in identifying subclinical LRLN. ${ }^{3}$ It should also be noted that Ortner's syndrome may manifest due to associated pulmonary hypertension or left ventricular dysfunction in severe mitral stenosis. Although the data for symptom improvement following valvular intervention is excellent (especially in those which valvular orifice area improves), there are few data at present to conclude that regression of left atrial enlargement and pulmonary arterial hypertension with symptoms are associated with Ortner's syndrome. ${ }^{1}$

\section{Conclusion}

Although rare, mitral stenosis can present clinically as Ortner's syndrome. It remains pertinent for clinicians to be aware of clinical features linked to mitral stenosis including its more uncommon presentations, such as in our case, as earlier intervention may greatly improve prognosis.

\section{References}

1 Chandrashekhar Y, Westaby S, Narula J. Mitral stenosis. Lancet 2009;374:1271-83.

2 Bickle IC, Kelly BE, Brooker DS. Ortner's syndrome: a radiological diagnosis. Ulster Med J 2002;71:55-6.

3 Subramaniam V, Herle A, Mohammed N, Thahir M. Ortner's syndrome: case series and literature review. Braz J Otorhinolaryngol 2011;77:559-62.

4 Escribano JF, Carnés J, Crespo MA, Antón RF. Ortner's syndrome and endoluminal treatment of a thoracic aortic aneurysm: a case report. Vasc Endovascular Surg 2006:40:75-8.

5 Hermans C, Manocha S, McLaughlin JE, Lipman M, Lee CA. Ortner syndrome and haemophilia. Haemophilia 2005;11:548-51.

6 Wunderlich NC, Dalvi B, Ho SY, Küx H, Siegel RJ. Rheumatic mitral valve stenosis: diagnosis and treatment options. Curr Cardiol Rep 2019;21:14.

Address for correspondence: Dr Raja EF Raja Shariff, Universiti Teknologi Mara Sungai Buloh, Jalan Hospital, 47000 Sungai Buloh, Selangor, Malaysia.

Email: rajaezman@gmail.com 